\title{
Changing climatic response: a conceptual model for glacial cycles and the Mid-Pleistocene Transition
}

\section{Daruka ${ }^{1}$ and P. D. Ditlevsen ${ }^{2}$}

${ }^{1}$ Johannes Kepler University, Institute of Semiconductor and Solid State Physics, Altenbergerstrasse 69, 4040 Linz, Austria

${ }^{2}$ Centre for Ice and Climate, Niels Bohr Institute, University of Copenhagen, Juliane Maries Vej 30, 2100 Copenhagen, Denmark

Received: 4 February 2014 - Accepted: 18 February 2014 - Published: 13 March 2014

Correspondence to: I. Daruka (istvan.daruka@jku.at) and P. D. Ditlevsen (pditlev@nbi.ku.dk) Published by Copernicus Publications on behalf of the European Geosciences Union.

\section{Changing climatic response \\ I. Daruka and \\ P. D. Ditlevsen}

\section{Title Page}

\section{Abstract}

Conclusions

Tables

Figures

14

4

Back

\section{Full Screen / Esc}

Printer-friendly Version

Interactive Discussion 


\section{Abstract}

Milankovitch's astronomical theory of glacial cycles, attributing ice age climate oscillations to orbital changes in Northern Northern-Hemisphere insolation, is challenged by the paleoclimatic record. The climatic response to the variations in 5 insolation is far from trivial. In general the glacial cycles are highly asymmetric in time, with slow cooling from the interglacials to the glacials (inceptions) and very rapid warming from the glacials to the interglacials (terminations). We shall refer to this fast-slow dynamics as the "saw-tooth" shape of the paleoclimatic record. This is non-linearly related to the time-symmetric variations in the orbital forcing. However, the

(MPT) occurring about one million years ago. During that event, the prevailing $41 \mathrm{kyr}$ glacial cycles, corresponding to the almost harmonic obliquity cycle were replaced by longer saw-tooth shaped cycles with a time scale around $100 \mathrm{kyr}$. The MPT must have been driven by internal changes in climate response, since it does not correspond to any apparent changes in the orbital forcing. In order to identify possible mechanisms causing the observed changes in glacial dynamics, it is relevant to study simplified models with the capability of generating temporal behavior similar to the observed records. We present a simple oscillator type model approach, with two variables, a temperature anomaly and an ice volume analogous, climatic memory term.

20 The generalization of the ice albedo feedback is included in terms of an effective multiplicative coupling between this latter climatic memory term (representing the internal degrees of freedom) and the external drive. The simple model reproduces the temporal asymmetry of the late Pleistocene glacial cycles and suggests that the MPT can be explained as a regime shift, aided by climatic noise, from a period 1 frequency 25 locking to the obliquity cycle to a period 2-3 frequency locking to the same obliquity cycle. The change in dynamics has been suggested to be a result of a slow gradual decrease in atmospheric greenhouse gas concentration. The presence of chaos in

\section{CPD}

10, 1101-1127, 2014

Changing climatic response

I. Daruka and

P. D. Ditlevsen

\section{Title Page}

Abstract

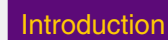

Conclusions

Tables

References

Figures

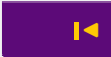

4

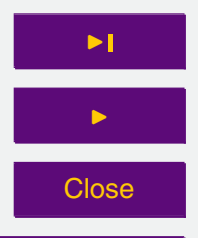

Full Screen / Esc

Printer-friendly Version

Interactive Discussion 
the (non-autonomous) glacial dynamics and a critical dependence on initial conditions raises fundamental questions about climate predictability.

\section{Introduction}

The Pleistocene climate record is more-or-less synonymous with the global stacked 5 marine core benthic foraminiferal $\delta^{18} \mathrm{O}$ record (Shackleton, 1997; Lisiecki et al., 2005), which is a combined proxy for global ice volume and ocean temperature. Single core deviations from the stack can to some extent be interpreted as local climate variations, however, with a poor signal-to-noise ratio due to bioturbation. The stacked record correlates strongly with the Antarctic ice core isotope records (Petit et al., 10 1999; Augustin et al., 2004) for the past 420 and $800 \mathrm{kyr}$, which thus provides limited additional information. The challenge is therefore to discriminate between possible models for explaining the connection between the insolation and the global mean climatic response represented by the stacked marine isotope record.

Milutin Milankovitch, whose seminal contribution to ice age theory was to calculate 15 the orbital parameters and the insolation, considered (together with climatologist Wladimir Köppen) the mid- to high Northern latitude summer insolation to be most strongly determining glacial melt and thus the waxing and waning of the ice sheets. The $65^{\circ} \mathrm{N}$ summer solstice insolation has thus been canonized as the "Milankovitch forcing" (Berger, 2012). However, this particular component of the insolation is most strongly 20 influenced by the approximately $20 \mathrm{kyr}$ precessional cycle, whereas the paleoclimatic record shows that the response is strongest in the 41 and $100 \mathrm{kyr}$ bands. The glacial melt depends rather on the integrated summer insolation (as an indicator of positive degree days) than on the summer solstice insolation. The integrated summer insolation is indeed dominated by the $41 \mathrm{kyr}$ obliquity cycle (Huybers, 2006). This compares well 25 with the climate record prior to the MPT $41 \mathrm{kyr}$ world, while the late Pleistocene $100 \mathrm{kyr}$ world compares more with the variations in the eccentricity of the orbit. The changes in insolation due to changes in eccentricity are an order of magnitude smaller than the

\section{CPD}

$10,1101-1127,2014$

Changing climatic response

I. Daruka and

P. D. Ditlevsen

\section{Title Page}

Abstract

Conclusions

Tables

References

Figures

14

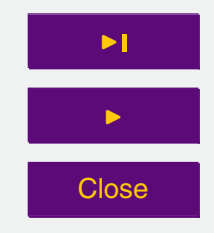

Back

\section{Full Screen / Esc}

Printer-friendly Version

Interactive Discussion 
changes due to precession and obliquity. This is referred to as the $100 \mathrm{kyr}$ problem of the Milankovich theory (Imbrie et al., 1993). The solution to this problem is probably that the $100 \mathrm{kyr}$ world is not paced by eccentricity, which is also why the $400 \mathrm{kyr}$ modulation to the eccentricity is not seen in the climatic response. This is referred to as the $400 \mathrm{kyr}$ 5 problem or the Stage 11 (MIS 11) problem: marine isotope stage 11 should not have been an interglacial if changes in eccentricity were the driver. It has been suggested that the $100 \mathrm{kyr}$ world could rather be seen as multiplets of the $41 \mathrm{kyr}$ obliquity cycle, such that they are approximately 80 and $120 \mathrm{kyr}$ long, occurring in a more or less alternating way (Huybers, 2007; Ditlevsen, 2009). Note in Fig. 1 that the duration 10 between the warm states around $200 \mathrm{kyrBP}$ (MIS 7 and MIS 6) is approximately $40 \mathrm{kyr}$.

Since there are no apparent changes in the astronomical forcing at the MPT, the transition must be governed by the internal dynamical response to the forcing. The hypothesis is that a gradual change in some environmental parameter of the system led to a dynamical change in the response to the orbital forcing. Two main hypothesis 15 have been put forward for the change in environment; either a slow decrease in atmospheric $p \mathrm{CO}_{2}$ (Saltzman and Maasch, 1991) led to reduced greenhouse warming and the possibility of deeper glaciations, or slow glacial erosion of the regolith under the glaciers, such that the glaciers after the MPT would grow on the bedrock, which permits higher glaciers to be stable (Clark and Pollard, 1998). For a review see Clark et al. 20 (2006). Here we shall argue by introducing a semi-conceptual driven climate oscillator model that the MPT could be a result of frequency locking to the orbital forcing, such that a slight change in parameters can alter the period of the frequency locking.

The phenomenon of phase-locking for explaining climatic response to orbital forcing has been suggested before. De Saedeleer et al. (2013) discuss in terms of the Van der Pol oscillator the possibility of synchronizing to spectral components of the $65^{\circ} \mathrm{N}$ summer solstice insolation. These are expressed in terms of a d'Alembert series resulting from the perturbative calculation of the orbital parameters (Berger, 1978). Here we are not so much concerned with the problem of which component of the orbital forcing the climate system is most sensitive. Since the different orbital parameters

\section{CPD}

10, 1101-1127, 2014

Changing climatic response

I. Daruka and

P. D. Ditlevsen

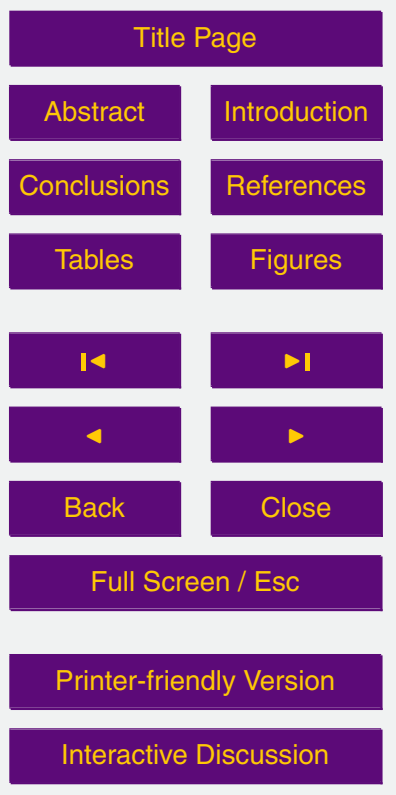


influence the insolation very differently in both seasonality and latitudinal variation it is unlikely that a single time series (with annual or less resolution) is sufficient to give the full account of the Pleistocene glacial cycles.

Still it is an open problem to which extent the global stack marine isotope record 5 itself is sufficient to discriminate between the suggested low dimensional or conceptual models of the Pleistocene glacial cycles. A decisive model of the MPT, probably have to wait for future comprehensive climate model simulations and strong improvements in the regional climate reconstructions. Exploring alternative mechanisms in simple and conceptual models are thus important both for identifying robust features and for

\section{Existing models}

In order to put our model in perspective, we review in the following some of the proposed models and their limitations. This is by no means a comprehensive review. The models of glacial cycles can roughly be categorized in two types: firstly, in mono15 or multi-stable state models where the periodicity is solely a result of the periodicity of the orbital forcing. Secondly, in climate oscillator models where the glacial cycles in one way or another result from the internal oscillator resonating with the orbital forcing. For a thorough review of oscillator type models see Crucifix (2012).

The classical energy balance models (Budyko, 1969; Sellers, 1969) belong to 20 the first category. In these models the ice-albedo feedback results in a two-state system, a present climate state and a glaciated state, which today is considered more realistically to describe the Snowball Earth climate. The relative weakness in the magnitude of the $100 \mathrm{kyr}$ eccentricity cycle in insolation led to the introduction of the concept of stochastic resonance (Benzi et al., 1982).

25 For contrasting the glacial dynamics before and after the MPT a semi empirical model was proposed by Paillard (1998). The model is a rule based threshold model with three possible stable climate states, an interglacial, a mild glacial and a full glacial. With

\section{CPD}

10, 1101-1127, 2014

Changing climatic response

I. Daruka and

P. D. Ditlevsen

\section{Title Page}

Abstract Introduction

Conclusions

Tables

References

Figures

14

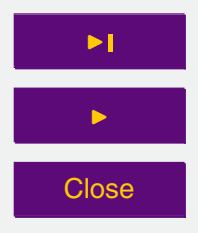

Back

\section{Full Screen / Esc}

Printer-friendly Version

Interactive Discussion 
specific rules of transitions and a slow change of threshold, the model reproduces the Pleistocene record including the MPT as a response to the Milankovitch forcing. An empirical dynamical model, with a three stable state bifurcation diagram, explaining the specific rules of transitions in the Paillard model has been proposed (Ditlevsen, 5 2009). In this model a change of the bifurcation structure causes the MPT. A different approach was taken in Huybers and Wunsch (2005) for explaining the $100 \mathrm{kyr}$ world as a result of the $41 \mathrm{kyr}$ orbital forcing. This is a stochastic threshold model with a linear drift towards glaciation. When a threshold proportional to the obliquity is reached the glaciation terminates and the climate is reset to the interglacial condition. This model 10 naturally reproduces the sawtooth shape of the climate curve. The linear drift assumes a very long internal time scale of the order $100 \mathrm{kyr}$ for glaciation. By a simple rescaling of this time scale the model can also reproduce the $41 \mathrm{kyr}$ world, however, with more time asymmetric (sawtooth shaped) glaciations, than observations indicate.

Many different types of oscillator models have been suggested: The dynamics of ice 15 sheets is roughly: Higher temperature $\rightarrow$ higher accumulation $\rightarrow$ growing ice sheet $\rightarrow$ high albedo $\rightarrow$ lower temperature (Kallen et al., 1979; Tziperman and Gildor, 2003). This results in free oscillations of the order $5-15 \mathrm{kyr}$, which are to fast to account for the glacial cycles. The non-linear response in this model to beat periods (combination tones) between the 19 and the $23 \mathrm{kyr}$ precessional frequencies has been suggested to explain the $100 \mathrm{kyr}$ glacial cycles (Le Treut and Ghil, 1983). To obtain free climate oscillations of as long a duration as $100 \mathrm{kyr}$, the combined effect of isostatic rebound and reduced accumulation with a high ice sheet (the elevation dessert effect) is another suggestion (Hyde and Peltier, 1985). None of these models attempt to explain the MPT shift in glacial periods. It was suggested by Saltzman and Maasch (1991) that the ice sheet growth is controlled by the deep-ocean temperature: low ocean temperature $\rightarrow$ higher uptake of atmospheric $\mathrm{CO}_{2} \rightarrow$ less greenhouse warming $\rightarrow$ growth of ice sheets and sea ice $\rightarrow$ reduced meridional oceanic heat transport $\rightarrow$ higher ocean temperature. In the model this is a limit cycle, which is initiated at the MPT through a Hopf bifurcation as a result of a slow decrease in $\mathrm{CO}_{2}$ from increased weathering.

\section{CPD}

$10,1101-1127,2014$

Changing climatic response

I. Daruka and

P. D. Ditlevsen

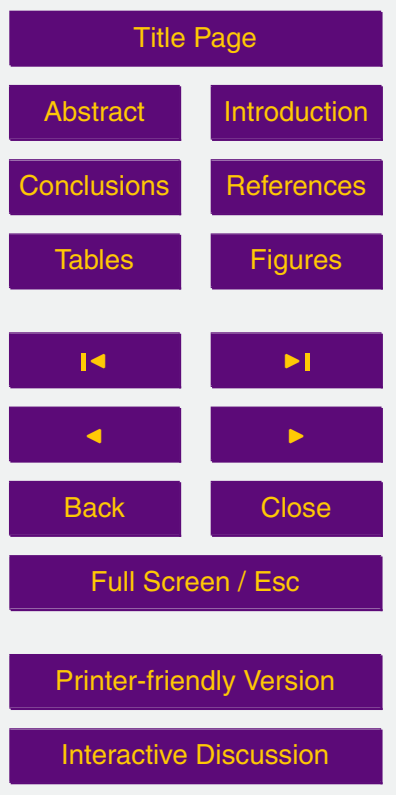


This model is not concerned with the $41 \mathrm{kyr}$ climate oscillations prior to the MPT. A switch mechanism involving the sea-ice was proposed by Gildor and Tziperman (GT) (Tziperman and Gildor, 2003). In this GT-model the feedback loop is: high seaice cover (limited by the warm mid-latitude ocean) $\rightarrow$ reduced atmospheric temperature 5 and precipitation (accumulation) over the ice sheets $\rightarrow$ negative mass balance $\rightarrow$ rapid retreat of the ice sheets $\rightarrow$ reduced albedo $\rightarrow$ increased temperature $\rightarrow$ rapid retreat of the sea-ice $\rightarrow$ increased precipitation and a positive mass balance. This is a relaxation oscillator, where the $100 \mathrm{kyr}$ cycle is internally driven and independent of the orbital forcing. The time scale for growth of the ice sheets estimated as $V /$ Acc, where $V$ is 10 the volume of the ice sheet and Acc is the accumulation (in suitable units) is of the order $10-30 \mathrm{kyr}$. The time scale of $100 \mathrm{kyr}$ glacial cycles in the GT-model is rather estimated from $V /(A c c-A b l)$, where $(A c c-A b l)$ is the difference between accumulation and ablation in the growth phase. In principle this estimate is not well constrained, since in the case of almost mass balance this growing time is infinite. If the deep ocean in 15 the model is warm enough, the sea-ice switch is not active, and the model oscillates linearly with the orbital forcing. Gradual cooling of the deep ocean, crossing a threshold at MPT activates the sea-ice switch mechanism and the $100 \mathrm{kyr}$ oscillations of the late Pleistocene period. Alternatively, the $41 \mathrm{kyr}$ world could be self-sustained oscillations, perhaps locked to the obliquity cycles (Ashkenazy and Tziperman, 2004).

All the above suggested physical mechanisms are potentially at play in the climate system, thus it is difficult to assess the relative importance without realistic quantitative modelling, which at present is computationally prohibited. However, identifying possible dynamical mechanisms for explaining the observed record merits the more conceptual model approaches. Rial (2004) suggested a logistic-delayed differential equation for the ice volume coupled, through a carrying capacity, to a temperature. The temperature is determined by energy balance between incoming and outgoing radiation. By changing the coupling via the carrying capacity, the MPT is reproduced. The underlying physical mechanism is difficult to identify, partly because delay-equations are notoriously difficult to analyse. An even simpler approach was taken by Huybers (2009), in which the

\section{CPD}

10, 1101-1127, 2014

Changing climatic response

I. Daruka and

P. D. Ditlevsen

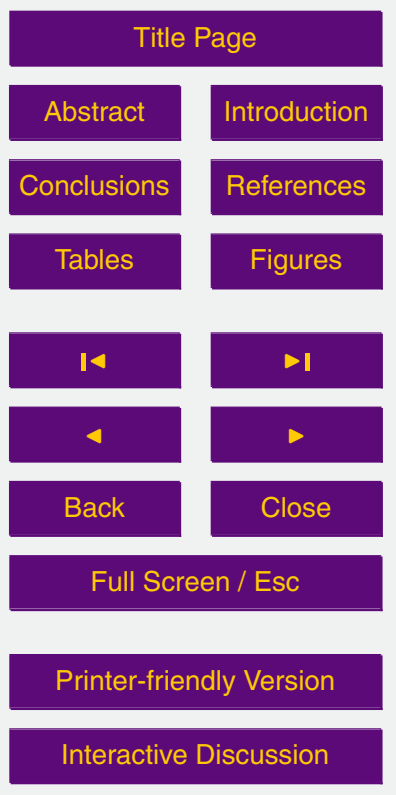


Pleistocene climate is described by a discrete map, where the ice volume depends on the ice volume with a lag of $9 \mathrm{kyr}$, which is approximately the lag between climate oscillations and their derivative in a $40 \mathrm{kyr}$ harmonic cycle. The hypothesis is that the late Pleistocene cycles are purely chaotic, while the $41 \mathrm{kyr}$ cycle prior to the MPT are 5 results of coincidental oscillations near an unstable period 2 cycle in the map. However, within the model framework, the observed long sequence of $41 \mathrm{kyr}$ cycles prior to the MPT seems highly unlikely.

The conceptual modelling is also our approach here, where we shall argue that the MPT could be a change in the internal dynamics leading to a change in frequency 10 locking to the obliquity cycle. This would imply a fundamental limitation in climate predictability, such as inferences about the next inception. We do, however, find this type of un-falsifiable very long time predictions into the future rather academic, but the suggested critical dependence on model parameters is potentially an important guideline for more realistic future model simulations and theories of the Pleistocene 15 glacial dynamics.

\section{Model}

As a minimal modelling approach we explore a two variable non-linear oscillator model, containing a scaled temperature anomaly $\tau=\left(T-T_{0}\right) / T_{0}$, where $T$ is a large scale (Northern Hemisphere or global) mean surface temperature, and $T_{0}$ is the long term

mean. The other variable $x$ was inspired by the the global ice mass anomaly. However, the approaches of Saltzman and Maasch (1991) and that of Tziperman and Gildor (2003) clearly indicate that besides the ice volume, also the deep sea temperature and possible other factors play a decisive role in determining the internal climate dynamics, leading to coupled and nonlinear climatic memory effects. It is possible that if two (or possess a unique characteristic time, and also the climatic state dependent effective internal timescale might be much longer than any of the involved characteristic times

\section{CPD}

10, 1101-1127, 2014

Changing climatic response

I. Daruka and

P. D. Ditlevsen

\section{Title Page}

Abstract

Conclusions

Tables

References

Figures

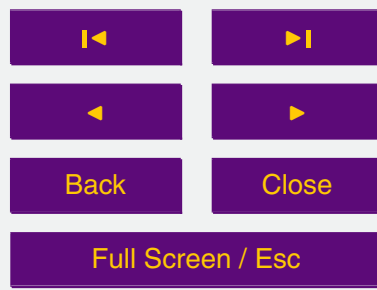

Printer-friendly Version

Interactive Discussion 
due to the potential multiplicative coupling. With the integral variable $x$ we mimic such integrated climatic memory effects.

In the light of the above, we consider a simple integrative relation between the effective climatic memory term $x$ and the temperature anomaly

${ }_{5} \quad \dot{x}(t)=\lambda \tau(t)$,

where $\lambda^{-1}$ represents the timescale unit of the climatic memory. The paleoclimatic record strongly indicate that the climate can be in one of more possible stable states. Within a two variable model, not resolving this multi-state dynamics, the evolution of the temperature anomaly is represented by an effective climate potential $V(x)$ which could 10 possess different local minima and a damping of the anomaly $(-\kappa \tau)$. The interaction between the internal dynamics and the external drive is modeled as a multiplicative coupling between the solar insolation $A(t)$ and the climatic memory term $x(t)$ :

$\dot{\tau}(t)=-V^{\prime}(x(t))-\kappa \tau(t)-x(t) A(t)+\sigma \eta(t)$,

where ' denotes derivative with respect to the argument. The last term is a climatic 15 noise term to account for unresolved processes, where $\eta(t)$ represents a white noise contribution with $\left\langle\eta(t) \eta\left(t^{\prime}\right)\right\rangle=\delta_{t, t^{\prime}}$.

Furthermore, we define

$V(x)=\alpha x-x^{2} / 2+x^{4} / 4$

as the effective climate potential. This is the simplest non-trivial polynomial multiple state potential, which can contain two minima representing two distinct stable climate states. We note that the non-symmetric, non-harmonic nature of the implemented climate potential renders no characteristic timescale to the climatic response. Furthermore, note that this potential is not the potential derived from the classical Budyko-Sellers energy balance model (Budyko, 1969), now believed to describe the Snowball Earth dynamics. The energy balance model includes the ice albedo effect in

CPD

10, 1101-1127, 2014

Changing climatic response

I. Daruka and

P. D. Ditlevsen

\section{Title Page}

Abstract Introduction

Conclusions

Tables

References

Figures

14

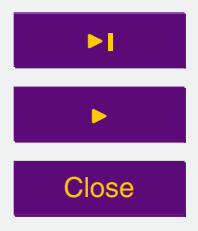

Back

\section{Full Screen / Esc}

Printer-friendly Version

Interactive Discussion 
the potential, while we include it in the forcing term $-x(t) A(t)$ involving a multiplicative coupling.

The model described by Eqs. (1)-(3) is a forced non-linear oscillator, including a multiplicative coupling between the internal dynamics (climatic memory effects) and 5 the external forcing and can possess a two state effective climatic potential (Eq. 2).

We will however not be concerned with the strongly non-linear regime, where multiple steady states are possible. On the contrary, we will throughout the rest of the paper use $\alpha=0.8$ in Eq. (2), thus $V(x)$ is a non-harmonic skewed potential with a single minimum. The major effect of the non-harmonic nature of the potential is that the frequency of 10 free oscillations (in case of no damping and no forcing) depends on the amplitude of oscillation. Thus the system does not have a natural internal frequency of oscillation.

The external drive has a period (time-scale) $T_{\text {ext }}=2 \pi / \omega$, where $\omega$ is the dominant frequency of the orbital forcing. In this work we shall not so much be concerned with the multi-frequency nature of the orbital forcing (De Saedeleer et al., 2013). The orbital changes in insolation strongly depends on latitude and season, which is not directly incorporated in an effective low dimensional model as presented here.

In the following we demonstrate that the model is capable of reproducing the climate record including the MPT and the time asymmetry, the sawtooth shapes, of the glacial cycles in the $100 \mathrm{kyr}$ world solely as a response to the obliquity cycles. In order 20 to explore the dynamical features of the model, we shall first simplify by applying a pure sinusoidal forcing $A(t)=A \sin (\omega t)$ with a period of $41 \mathrm{kyr}$, which quite accurately represents the obliquity cycle.

The simple model presented here exhibits surprisingly complex dynamics. Our postulate is that the MPT is a result of a slow environmental change represented in 25 a slow change in a model parameter. Here we restrict ourselves to changes in the climatic damping coefficient $k$. This change results in a shift from a period-1 frequency locking to a period 2 and 3 frequency locking. Such a change in the dynamical response to the forcing results in a very long non-periodic transient response, which might explain why the timing of the MPT varies so much (from 1.2 Myr to $800 \mathrm{kyr}$ ).

CPD

$10,1101-1127,2014$

Changing climatic response

I. Daruka and

P. D. Ditlevsen

\section{Title Page}

Abstract

Conclusions

Tables

References

Figures

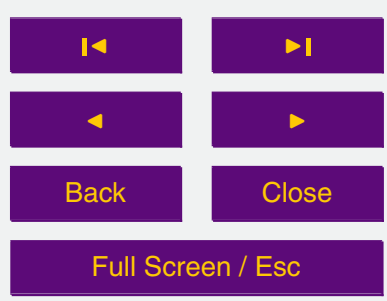

Printer-friendly Version

Interactive Discussion 


\section{Basic properties of the model}

In spite of the apparent simplicity of this conceptual model, it contains a rich dynamics in its basic mathematical and physical aspects. To illustrate this, first we apply a simple deterministic $(\sigma=0)$ sinusoidal external driving; $A(t)=A \sin (\omega t)$, taking $\omega=$ $52 \pi(42 \mathrm{kyr})^{-1}$ corresponding to the obliquity cycle. With this choice, the free parameters of the model are: $\alpha, A, k$ and $\lambda$. Again, we use the value $\alpha=0.8$, corresponding to a climatic potential $(V)$ with a single minimum. Also, if not stated otherwise, the parameter $\lambda$ was set to 10 (in appropriate units; $(100 \mathrm{kyr})^{-1}$ ) throughout our investigations, corresponding to a characteristic timescale of $10 \mathrm{kyr}$. The Eqs. (1)(3) were numerically integrated using a fourth-order Runge-Kutta scheme. As initial conditions, $\tau_{0}=0$ and $x_{0}=-1.2756$ were chosen. The latter value corresponds to the minimum of the climatic potential for $\alpha=0.8$.

We found the following types of temperature anomaly oscillations: linear response, the response frequency coincides with that of the driving; period doubling, period multiplication, with related "exotic" shaped oscillations; and chaotic behavior.

The period multiplication scenario is shown in Fig. 2 for a section of the $(A-k)$ parameter space. A standard procedure for identifying periodicity as multiple of the driving period, would be generating a Poincare map, and counting the number of intersections, regarding points within circles of some small radius $\epsilon$ as identical. Here we apply a slightly more robust method where the periodicity $n$ is calculated as

$$
n=\min _{m}: \int_{T_{\text {end }}-m T}^{T_{\text {end }}}|x(t)-x(t-m T)| \mathrm{d} t<\epsilon .
$$

Figure 2 demonstrates the occurrence of all sorts of periods and a period doubling route to chaos. We also note, that due to the existence of sharp phase boundary lines separating different period oscillations, a small change in the model parameters can lead to a remarkable transition in the climatic response. As an example, Fig. 3
CPD

10, 1101-1127, 2014

Changing climatic response

I. Daruka and

P. D. Ditlevsen

\section{Title Page}

Abstract Introduction

Conclusions

Tables

References

Figures

14

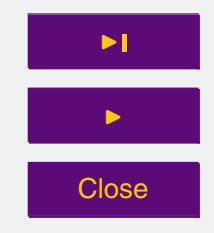

Back

Full Screen / Esc

Printer-friendly Version

Interactive Discussion 
demonstrates a sharp period doubling transition in the climatic response $\tau$ due to a very small, gradual change in the sinusoidal drive, which we otherwise consider constant in this paper. This way, the response to a slight change in the amplitude of the forcing results not only in a change in periodicity, but also in a change in the amplitude of the 5 response.

We found that in general, the appearance of chaos was promoted at small values of $\omega$, large values of $A$, large values of $\lambda$, and small values of $k$. Figure 4 shows the Lyapunov-exponent for the temperature anomaly (defined as $\partial \ln (\delta \tau) / \partial t$ ) for a section of the $(\lambda-\kappa)$ phase space where chaotic behavior occurs. Interestingly, while in certain 10 directions the Lyapunov exponent changes continuously from zero to a positive value, in other directions one observes an abrupt change.

The systematic mathematical investigation of the different period multiplied phases and the features of of chaos in the current model points beyond the scope of the present paper and will be reported elsewhere. Instead, we focus on the climatic relevance of 15 the possible nonlinear responses.

\subsection{Possible mechanism for the Mid-Pleistocene Transition}

The paleoclimatic record contains enough enigmas to realize that the Milankovitch theory of orbitally forced ice age cycles still has missing links. Especially explaining the MPT is a challenge. The $100 \mathrm{kyr}$ time scale is very long in comparison to reasonable estimates for internal climate oscillators to be at play. In the very inspiring paper (De Saedeleer et al., 2013) it was recently proposed, based on a study of the Van de Pol oscillator, that the climatic response to orbital forcing is a phase locking of internal periods to periods of the orbital forcing. This was proposed as a way of assessing the determining periods of the multi period orbital forcing. Here we demonstrate not have any internal periods of oscillation. This is a simple consequence of the fact that the proposed "climate potential" is not harmonic, thus the frequency of oscillation depends continuously on the amplitude of oscillation. The system can thus resonate

CPD

10, 1101-1127, 2014

Changing climatic response

I. Daruka and

P. D. Ditlevsen

\section{Title Page}

Abstract Introduction

Conclusions

Tables References Figures

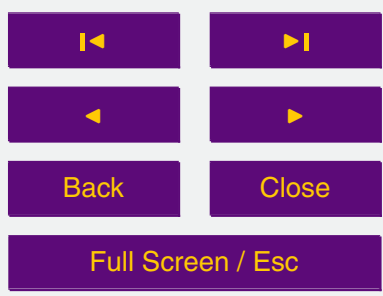

Printer-friendly Version

Interactive Discussion 
with the external forcing by adjusting the amplitude of the oscillation. Furthermore, as it was seen in the case of a simple harmonic forcing, by a slow change of parameters the system can show both period doubling transitions to a chaotic state and other transitions such as transition to period three response. We thus propose that

5 throughout the Pleistocene epoch, a gradual change in the climate system, which is represented by a slow variation in the model parameters took place. Such a change is feasible to assume as there is a long term decreasing trend in the global temperature of the Earth in the past $50 \mathrm{Myr}$ (with the exception of some isolated abrupt changes induced by extreme events related to volcanos, or sharp tectonic changes). This long 10 term global cooling can possibly be attributed to the gradual decrease of the global $\mathrm{CO}_{2}$ levels (Clark et al., 2006) and/or slow tectonic processes (Clark and Pollard, 1998). We may assume that the relevant driving is the $41 \mathrm{kyr}$ obliquity cycle throughout the Pleistocene epoch (Huybers and Wunsch, 2005; Huybers, 2009), in the latter part slowly varying parameters changed the dynamical response to periods two or three, 15 thus the last part of the Pleistocene climate is interpreted as a mixture of 80 and $120 \mathrm{kyr}$ responses to the obliquity cycle.

To illustrate this scenario we incorporate the assumed long-term change in the Earth's climatic response into the model by introducing a gradual decrease of the climatic damping coefficient $k$. In particular, we used the following implementation:

$20 \quad k(t)=\kappa_{1}+0.5\left(\kappa_{0}-\kappa_{1}\right)\left\{1.0-\tanh \left(\left(t-t_{0}\right) / t_{\mathrm{s}}\right)\right\}$,

where $\kappa_{0}>\kappa_{1}, t_{0}$, and $t_{\mathrm{s}}$ are positive constants.

Furthermore, we ran the model with the real obliquity data (Berger and Loutre, 1991), implementing $A(t)=4\left(O(t)-O_{\mathrm{av}}\right)$, where $O(t)$ is the actual obliquity dataset and $O_{\mathrm{av}}$ is its time average. Figure 1 shows a MPT that was simulated using model parameters $25 \kappa_{0}=1.1, \kappa_{1}=0.3, t_{0}=3.5 \mathrm{Myr}, t_{\mathrm{s}}=2 \mathrm{Myr}, \lambda=10$, and $x_{0}=-1.2756, \tau_{0}=0$ were used as initial conditions. One can see that the period of the oscillations changed from $41 \mathrm{kyr}$ to approximately $120 \mathrm{kyr}$ after the MPT.

CPD

$10,1101-1127,2014$

Changing climatic response

I. Daruka and

P. D. Ditlevsen

\section{Title Page}

Abstract

Conclusions

Tables

References

Figures

14

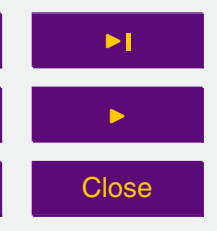

\section{Esc}

Full Screen / Esc

Printer-friendly Version

Interactive Discussion 
We also found that the maximum temperatures lagged $9 \mathrm{kyr}$ to the maximum amplitude of the external drive, throughout the whole Pleistocene epoch (Fig. 6). This result is in a good agreement with the findings of Huybers (2009).

Beside the change in response at the MPT the model also shows the characteristic 5 sawtooth shape of the oscillations after the MPT. The sawtooth shape is a typical signature of fast-slow dynamics. To further explore this feature of the model, Fig. 5 shows the details in the period three response to a sinusoidal forcing, repeating the third panel in Fig. 2. In the top panel of Fig. 5, the variables $\tau(t)$ (blue), $x(t)$ (green) and the forcing $A \sin (\omega t)$ (red) are shown. The grey bands indicate the rapid warnings 10 (fast phase), while the broad white sections indicate the gradual cooling periods (slow phase). What is important to notice is that the forcing (red curve) differs strongly for the two fast phase periods, while $x(t)$ is almost identical for the two periods. The product $x A \sin (\omega t)$ is shown in second panel, red curve. The model thus indicates that glacial terminations do not have to be in phase with the change in orbital forcing. The cause 15 of the fast transitions is solely encoded in the "potential drift" term $-\mathrm{d} V / \mathrm{d} x$, which is shown in the second panel, blue curve. To illustrate this further, a phase space portrait of the period three cycle is shown in the bottom left panel while the trajectory of the forcing vs. $x$ is shown in the middle panel. The coloring of the curves is such that the red part corresponds to the fast phases and the blue part to the slow phases. The fast-slow dynamics is thus a consequence of the asymmetry in the internal dynamics, represented in the simple model by the skewed climate potential, shown in the bottom right panel. Again the red part corresponds to the fast periods while the blue part corresponds to the slow periods. The obvious physical interpretation is that the melting and collapse of ice sheets and perhaps the melting of sea ice is a much faster process than the buildup of ice sheets from precipitation.

The glacial oscillations after the MPT are apparently much less regular than the $41 \mathrm{kyr}$ period one oscillations prior to the MPT. This could indicate either that the latter oscillations are such that they vary between a regime of period two and a regime of period three response. Or the response is simply a long transient response before

\section{CPD}

10, 1101-1127, 2014

Changing climatic response

I. Daruka and

P. D. Ditlevsen

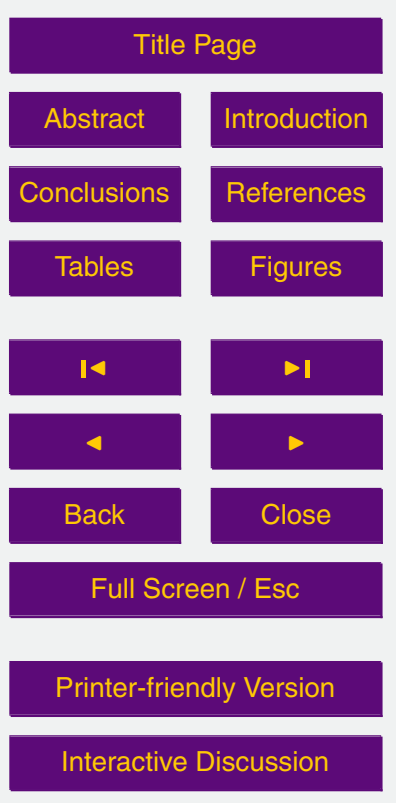

1114 
the system settles into a period three regime. This points to the fundamental issue of climate predictability, since the model shows both critical dependence on initial conditions and chaotic regimes. These are not synonymous, since the first can arise due to non-trivial boundaries for basins of attraction of different solutions even in the 5 non-chaotic regime.

To illustrate this, we implemented a small perturbation on the slow temporal change protocol of the climatic damping coefficient. We found that even such a small disturbance gets amplified and shifts a glacial by a whole obliquity cycle $(41 \mathrm{kyr})$ after $0.5 \mathrm{Myr}$ later, constituting a climatic butterfly effect (Fig. 7). This exponential amplification of a small perturbation is a sign of chaos in the system.

However, for this range of parameters, there is no chaos in the system when it is driven by a simple sinusoidal oscillation. To further investigate the underlying mechanism, instead of the real obliquity data, we forced the model with a simpler, two sinusoidal amplitude modulated sinusoidal oscillation:

${ }_{15} \quad A(t)=A_{0}\left\{1+A_{1} \sin \left(2 \pi t / T_{1}\right)+A_{2} \sin \left(2 \pi t / T_{2}\right)\right\} \sin \left(2 \pi t / T_{0}\right)$,

where we used $T_{0}=41 \mathrm{kyr}, T_{1}=212.24 \mathrm{kyr}, T_{2}=478.22 \mathrm{kyr}, A_{0}=2, A_{1}=0.2$, and $A_{2}=0.3$. In Eq. (4) we implemented $\kappa_{0}=2.1, \kappa_{1}=0.1, t_{0}=35 \mathrm{Myr}$, and $t_{\mathrm{s}}=2 \mathrm{Myr}$ as the adiabatic temporal change protocol parameters of the climatic damping coefficient. Furthermore, we set the parameter $\lambda=10$ and $x_{0}=-1.2756, \tau_{0}=0$ were used as initial 20 conditions. We found that even a small amount of climatic noise $\sigma=0.01$ in Eq. (2) can drastically change the behavior and can induce an MPT (Fig. 8a). Thus, this scenario corresponds to a noise aided MPT. As one can see, the late-Pleistocene climatic oscillations have two types, a $\sim 80 \mathrm{kyr}(\sim 2 \times 41 \mathrm{kyr})$ and a $\sim 120 \mathrm{kyr}(\sim 3 \times 41 \mathrm{kyr})$ period, following each other in an alternating way. This sequence gives a $100 \mathrm{kyr}$ period on average.

The increase of the modulation amplitudes in the above mentioned model external drive (Eq. 5) $\left(A_{1}=0.3\right.$, and $\left.A_{2}=0.8\right)$ and the implementation of a somewhat different damping coefficient temporal protocol (using $\kappa_{0}=1.1, \kappa_{1}=0.001, t_{0}=35 \mathrm{Myr}$, and $t_{\mathrm{s}}=$ 1115

\section{CPD}

10, 1101-1127, 2014

Changing climatic response

I. Daruka and

P. D. Ditlevsen

\section{Title Page}

Abstract

Conclusions

Tables

References

Figures

14

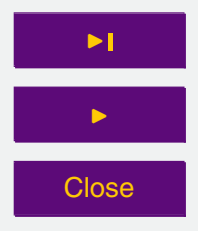

Back

\section{Full Screen / Esc}

Printer-friendly Version

Interactive Discussion 
2 Myr in Eq. 4) lead to the appearance of an MPT even in the absence of added noise. This behavior corresponds to an obliquity irregularities aided MPT scenario (Fig. 8b).

\section{CPD}

10, 1101-1127, 2014

\section{Conclusions}

We have formulated a semi-conceptual model to describe the Mid-Pleistocene 5 Transition, which we propose to be a period two and period three response to the $41 \mathrm{kyr}$ obliquity forcing. This resolves the "100 and $400 \mathrm{kyr}$ problems" of the Milankovitch theory, since the eccentricity cycle is proposed to be insignificant, and is omitted all together in the forcing. The change at the MPT is caused by a long term, gradual decrease of some parameter of the system. With the conceptual model approach, we 10 can only point to dynamical mechanisms and not to the real environmental change. However, the idea of a gradual change aligns with the decrease in global $\mathrm{CO}_{2}$ or tectonic re-arrangements as proposed in the literature.

The model does not in itself possess an internal frequency of oscillation, thus suggesting that the time scale of the glacial cycles is determined solely by the non15 linear response to the frequency of the obliquity pacing. We observe that the model also reproduces the observed saw-tooth shaped time reversal asymmetry observed in the record, which is not present in the forcing. In the model this is related to the larger amplitude of the glacial cycles after the MPT in comparison to the more symmetric cycles prior to the MPT. With the larger cycles, the system experience the non-linearity 20 in the internal dynamical response (the skewed climate potential in the model) much stronger than in the case of small, almost harmonic oscillations. As we have shown, the modulations in the forcing amplitude can also lead to a change in the periodicity of the response, rendering an intrinsically non-linear climatic behaviour.

Despite its simplicity, the model shows a surprisingly wide range of behaviours 25 depending on the forcing, the initial conditions and the values of the parameters. The observed variety of possible climatic responses in the model raises the perplexing question: How robust is the climatic history of the Earth? We are obviously not in the

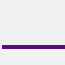

Changing climatic
response

P. D. Ditlevsen

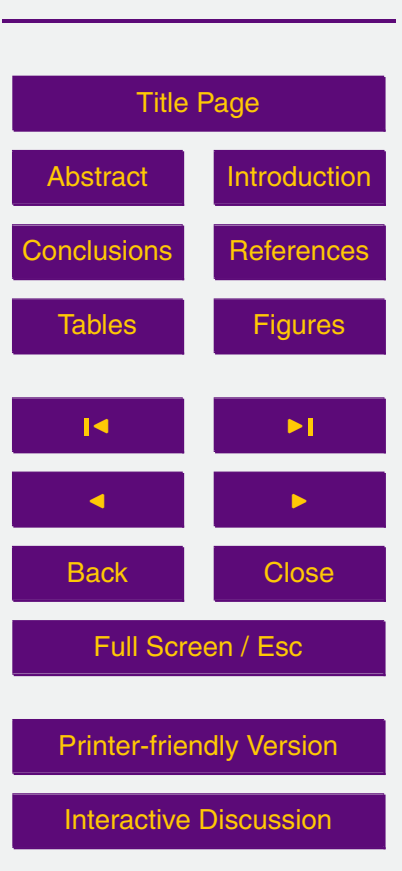

I. Daruka and 
position, where we can rerun the past. Thus we must ask in which sense, we should be able to model the past, by reproducing the evolution, which has been realised, or by reproducing the past in some statistical sense.

Furthermore, the presence of chaos in the present model suggests that this could 5 be manifested in the climate system itself in a so-called climatic butterfly effect: a small perturbation, or even just the internal climatic noise, might cause a significant shift in the glacial cycles. Obviously, this could impose fundamental limitations on long-term climate predictability.

Acknowledgements. I. Daruka acknowledges the support of the Hungarian Science Foundation under the contract OTKA NK72037 and that of the Hungarian Meteorological Service.

\section{References}

Augustin, L., Barbante, C., Barnes, P. R., and other EPICA community members: Eight glacial cycles from an Antarctic ice core, Nature, 429, 623-628, 2004. 1103

Ashkenazy, Y. and Tziperman, E.: Are the $41 \mathrm{kyr}$ oscillations a linear response to Milankovitch forcing?, Quaternary Sci. Rev., 23, 1879-1890, 2004. 1107

Benzi, R., Parisi, G., Sutera, A., and Vulpiniani, A.: Stochastic resonance in climatic change, Tellus, 34, 10-16, 1982. 1105

Berger, A. L.: Long-term variations of daily insolation and quaternary climatic changes, J. Atmos. Sci., 35, 2362-2367, 1978. 1104

Berger, A.: A brief history of the astronomical theories of paleoclimates, in: Climate Change, edited by: Berger, A., Mesinger, F., and Sijacki, D., Springer, Wien, 107-129, 2012. 1103

Berger, A. and Loutre, M. F.: Insolation values for the climate of the last 10 million of years, Quaternary Sci. Rev., 10, 297-317, 1991. 1113, 1120, 1126

Budyko, M. I.: The effect of solar radiation changes on the climate of the Earth, Tellus, 21, 611-619, 1969. 1105, 1109

Clark, P. U. and Pollard, D.: Origin of the middle Pleistocene transition by ice sheet erosion of regolith, Paleoceanography, 13, 1-9, 1998. 1104, 1113

Clark, P. U., Archer, D., Pollard, D., Blum, J. D., Rial, J. A., Brovkin, V., Mix, A. C., Pisias, N. G., and Roy, M.: The middle Pleistocene transition: characteristics, mechanisms,

\section{CPD}

10, 1101-1127, 2014

Changing climatic response

I. Daruka and

P. D. Ditlevsen

\section{Title Page}

Abstract

Introduction

Conclusions

References

Tables

Figures

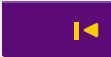

4

4

Back

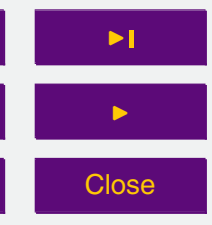

Full Screen / Esc

Printer-friendly Version

Interactive Discussion 
and implications for long-term changes in athmospheric $p \mathrm{CO}_{2}$, Quaternary Sci. Rev., 25, 3150-3184, 2006. 1104, 1113

Crucifix, M.: Oscillators and relaxation phenomena in Pleistocene climate theory, Philos. T. R. Soc. A, 370 1140-1165, 2012. 1105

5 De Saedeleer, B., Crucifix, M., and Wieczorek, S.: Is the astronomical forcing a reliable and unique pacemaker for climate? A conceptual model study, Clim. Dynam., 40, 273-294 2013, 2013. 1104, 1110, 1112

Ditlevsen, P. D.: Bifurcation structure and noise-assisted transitions in the Pleistocene glacial cycles, Paleoceanography, 24, PA3204, doi:10.1029/2008PA001673, 2009. 1104, 1106

10 Huybers, P.: Early Pleistocene glacial cycles and the integrated summer insolation forcing, Science, 313, 508-511, 2006. 1103

Huybers, P: Glacial variability over the last two million years: an extended depth-derived age model, continuous obliquity pacing, and the Pleistocene progression, Quaternary Sci. Rev., 26, 37-55, 2007. 1104

15 Huybers, P.: Pleistocene glacial variability as a chaotic response to obliquity forcing, Clim. Past, 5, 481-488, doi:10.5194/cp-5-481-2009, 2009. 1107, 1113, 1114

Huybers, P. and Wunsch, C.: Obliquity pacing of the late Pleistocene glacial terminations, Nature, 434, 491-494, 2005. 1106, 1113

Hyde, W. T. and Peltier, W. R.: Sensitivity experiments with a model of the ice age cycle. The response to harmonic forcing, J. Atmos. Sci., 42, 2170-2188, 1985. 1106

Imbrie, J., Berger, A., Boyle, E. A., Clemens, S. C., Duffy, A., Howard, W. R., Kukla, G., Kutzbach, J., Martinson, D. G., Mclntyre, A., Mix, A. C., Molfino, B., Morley, J. J., Peterson, L. C., Pisias, N. G., Prell, W. L., Raymo, M. E., Shackleton, N. J., and Toggweiler, J. R.: On the structure and origin of major glaciation cycles 2, the 100000 -year cycle, Paleoceanography, 8, 699-735, 1993. 1104

Kallen, E., Crafoord, C., and Ghil, M.: Free oscillations in a climate model with ice-sheet dynamics, J. Atmos. Sci., 36, 2292-2303, 1979. 1106

LeTreut, H. and Ghil, M.: Orbital forcing, climate interactions, and glacial cycles, J. Geophys. Res., 88, 5167-5190, 1983. 1106

30 Lisiecki, L. E. and Raymo, M. E.: A Pliocene-Pleistocene stack of 57 globally distributed benthic $\delta^{18} \mathrm{O}$ records, Paleoceanography, 20, PA1003, doi:10.1029/2004PA001071, 2005. 1103, 1120

\section{CPD}

10, 1101-1127, 2014

Changing climatic response

I. Daruka and

P. D. Ditlevsen

\section{Title Page}

Abstract

Introduction

Conclusions

References

Tables

Figures

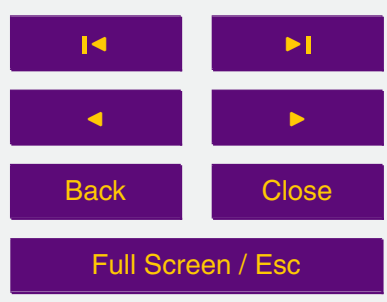

Printer-friendly Version

Interactive Discussion 
Paillard, D.: The timing of Pleistocene glaciations from a simple multiple-state climate model, Nature, 391, 378-381, 1998. 1105

Petit, J. R., Jouzel, J., Raynaud, D., Barkov, N. I., Barnola, J.-M., Basile, I., Benders, M., Chappellaz, J., Davis, M., Delayque, G., Delmotte, M., Kotlyakov, V. M., Legrand, M., $5 \quad$ Lipenkov, V. Y., Lorius, C., Pépin, L., Ritz, C., Saltzman, E., and Stievenard, M.: Climate and atmospheric history of the past 420,000 years from the Vostok ice core, Antarctica, Nature, 399, 429-436, 1999. 1103

Rial, J. A.: Abrupt climate change: chaos and order at orbital and millennial scales, Global Planet. Change, 41, 95-109, 2004. 1107

10 Saltzman, B. and Maasch, K.: A first-order global model of late Cenozoic climate change II: a simplification of $\mathrm{CO}_{2}$ dynamics, Clim. Dynam., 5, 201-210, 1991. 1104, 1106, 1108

Sellers, W. D.: A climate model based on the energy balance of the earth-athmosphere system, J. Appl. Meteorol., 8, 392-400, 1969. 1105

Shackleton, N. J.: Deep-sea sediment record and the Pliocene-Pleistocene boundary, Quartern. Int., 40, 33-35, 1997. 1103

Tziperman, E. and Gildor, H.: On the mid-Pleistocene transition to $100 \mathrm{kyr}$ glacial cycles and the asymmetry between glaciation and deglaciation times, Paleoceanography, 18, 1-8, 2003. 1106, 1107, 1108

\section{CPD}

10, 1101-1127, 2014

Changing climatic response

I. Daruka and

P. D. Ditlevsen

\section{Title Page}

\section{Abstract}

Introduction

Conclusions

References

Tables

Figures

14

4

Back

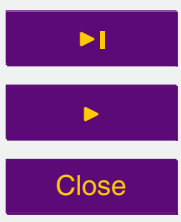

Full Screen / Esc

Printer-friendly Version

Interactive Discussion 


\section{CPD}

10, 1101-1127, 2014

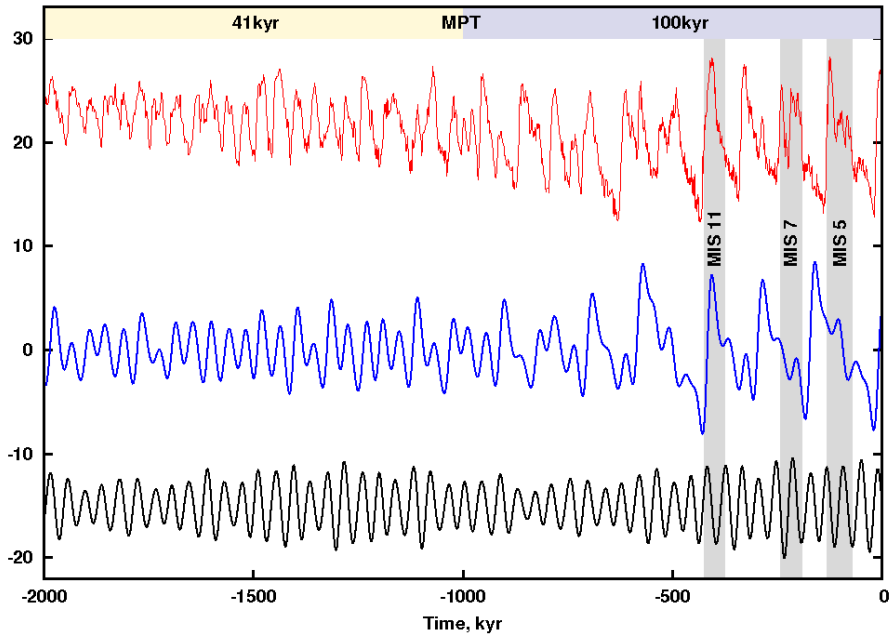

\section{Changing climatic response}

I. Daruka and

P. D. Ditlevsen

\section{Title Page}

Fig. 1. The Mid-Pleistocene Transition (MPT). The red curve corresponds to the composite deep sea foraminiferal isotope record (Lisiecki et al., 2005). The MPT is seen as a change around $1 \mathrm{MyrBP}$ in period from $41 \mathrm{kyr}$ in the early Pleistocene to approximately $100 \mathrm{kyr}$ in the late Pleistocene. The former cycles are symmetric, while the latter cycles are asymmetric ("sawtooth shaped"). The blue curve shows the model results for the related temperature anomaly when driven by real obliquity data (Berger and Loutre, 1991), represented by the black curve. Both the foraminiferal record and the obliquity data were scaled and shifted vertically for better visual representation. The parameter values used for this plot are discussed in the text.

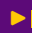

$\triangleright$

Close 

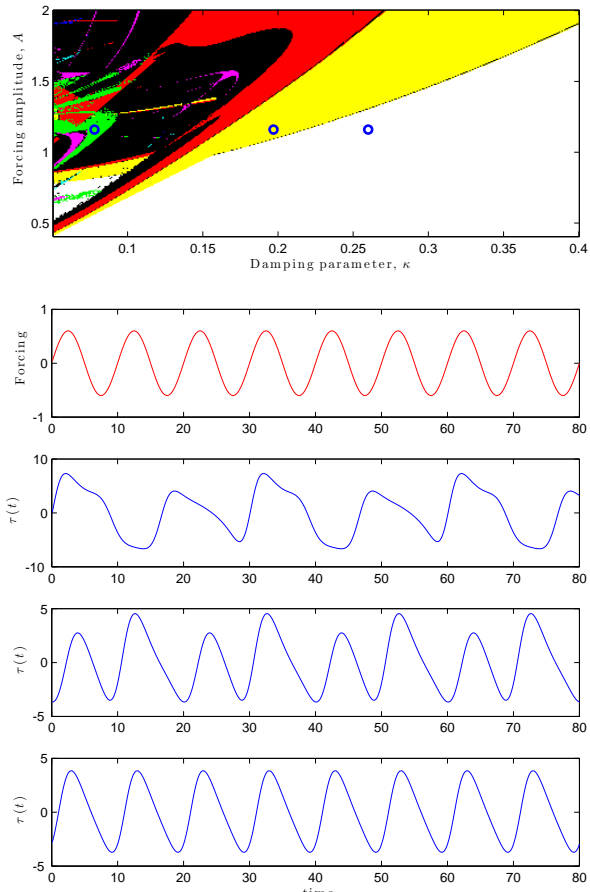

Fig. 2. The behavior of the model driven by a sinusoidal forcing $A \sin (\omega t)$, shown in red in the second panel. The top panel shows the periods in multiplets of the driving period $(2 \pi / \omega)$ : White corresponds to periodic solutions with period 1, yellow: period 2, green: period 3, red: period 4, magenta: period 6 , black: $\geq 8$, including non-periodic (chaotic) solutions. The bottom three panels show the solutions corresponding to the blue circles in the top panel left-to-right (periods 3, 2, 1 respectively). Changing parameters along a horizontal line shows a period doubling route to chaos. We used the parameters $\omega=2 \pi / 10, \lambda=0.1$, with $x_{0}=0$, and $\tau_{0}=1$ as initial conditions for this plot.

\section{CPD}

10, 1101-1127, 2014

Changing climatic response

\section{Daruka and}

P. D. Ditlevsen

\section{Title Page}

\section{Full Screen / Esc}

Printer-friendly Version

Interactive Discussion 


\section{CPD}

10, 1101-1127, 2014

\section{Changing climatic} response

I. Daruka and

P. D. Ditlevsen

\section{Title Page}

Fig. 3. Even a very small, visually unnoticeable gradual change in the sinusoidal driving (plotted in green) can lead to a sharp, remarkable period doubling in the climatic response $\tau$ (plotted in red). We used the parameters $\omega=7, \lambda=10, k=1.3$, and $A(t)=0.008 t \sin (\omega t)$ with $x_{0}=$ -1.2756 , and $\tau_{0}=0$ as initial conditions for this plot.

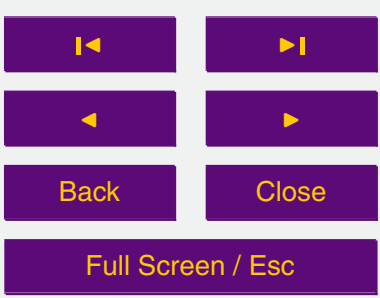

Printer-friendly Version

Interactive Discussion 


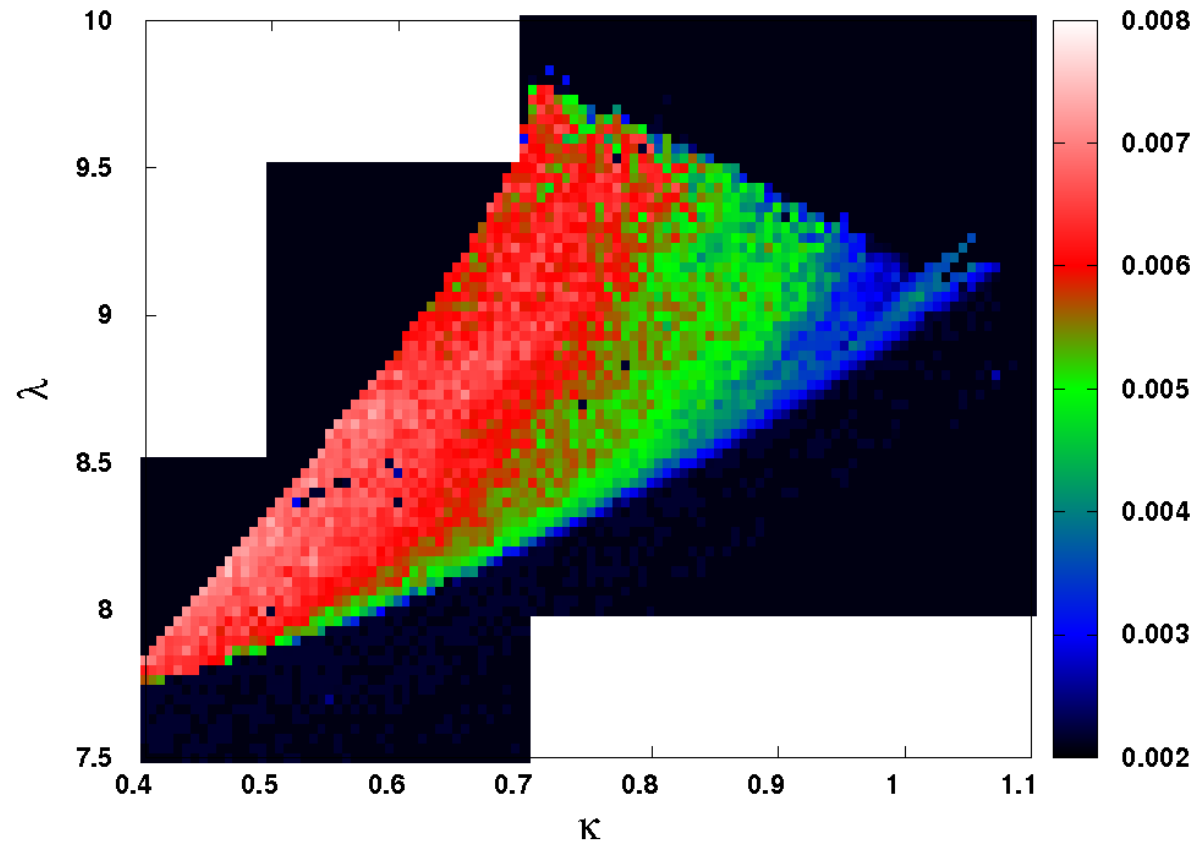

Fig. 4. Plot of the Lyapunov-exponent (in units of $\mathrm{kyr}^{-1}$ ) for the temperature anomaly $(\tau)$ oscillations for a section of the $(\lambda-k)$ parameter phase space, demonstrating the presence of chaos in the model. The parameter values are $\omega=10, A=1$, and $x_{0}=0, \tau_{0}=1$ were used as initial conditions.

\section{CPD}

10, 1101-1127, 2014

Changing climatic response

\section{Daruka and}

P. D. Ditlevsen

\section{Title Page}

\section{Abstract}

Introduction

Conclusions

References

Tables

Figures

14

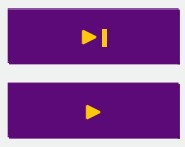

Back

Close

\section{Full Screen / Esc}

Printer-friendly Version

Interactive Discussion 


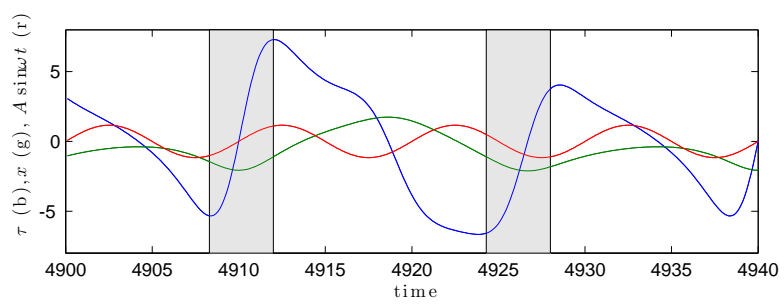

CPD

10, 1101-1127, 2014
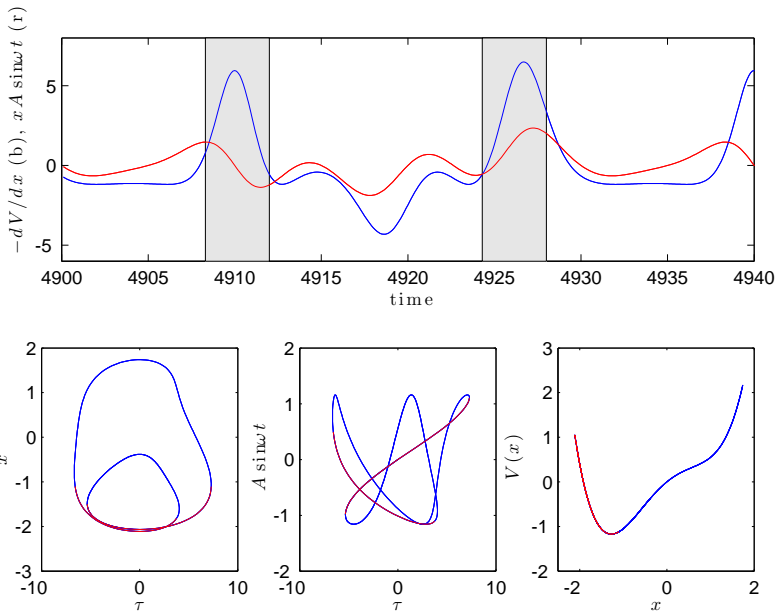

Fig. 5. The period three response to a sinusoidal forcing, as in Fig. 2. The parameter values are $\alpha=0.8, \lambda=0.087$, $\kappa=0.078, A=1.16, \omega=2 \pi / 10$. Top panel shows the variables $\tau(t)$ (blue), $x(t)$ (green) and $A \sin (\omega t$ ) (red). The grey bands with rapid increase in $\tau(t)$ are defined as the fast periods, while the rest are the slow periods. The middle panel shows the two dominant terms in the right hand side of the dynamical Eq. (2): $-\mathrm{d} V / \mathrm{d} x$ (blue) and $x A \sin (\omega t)$ (red). It is seen that the fast dynamics is governed by the former term, representing internal dynamics. The bottom left panel show the phase space portrait of the period three solution, while the bottom middle panel shows the forcing $A \sin (\omega t)$ vs. $\tau$. The bottom right panel shows the "climate potential". The asymmetry of the potential is responsible for the fast-slow dynamics and the saw-tooth shape of the record. The red parts of the curves correspond to the fast periods.

\section{Changing climatic response}

\section{Daruka and \\ P. D. Ditlevsen}

\section{Title Page}

\section{Full Screen / Esc}

Printer-friendly Version

Interactive Discussion 


\section{CPD}

10, 1101-1127, 2014

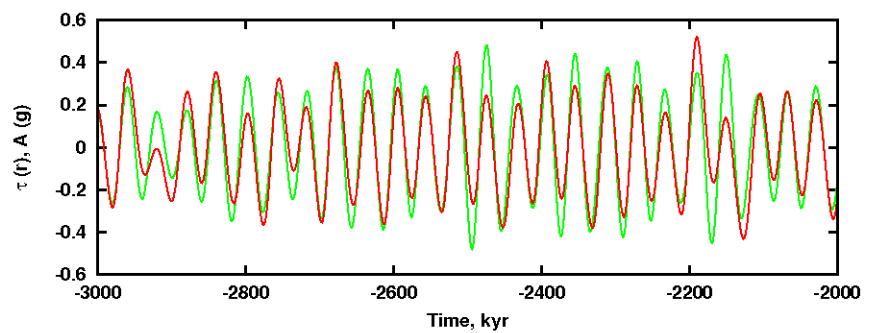

\section{(a)}

\section{Changing climatic response}

\section{Daruka and \\ P. D. Ditlevsen}

\section{Title Page}

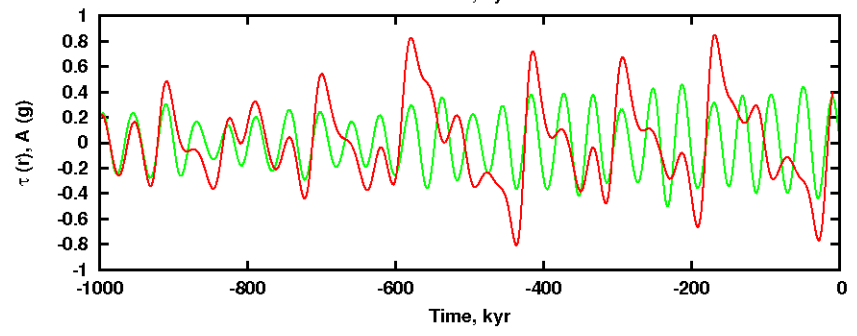

(b)

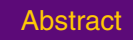

Introduction

Conclusions

References

Tables

Figures

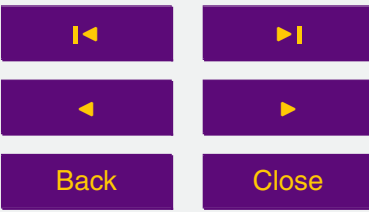

Full Screen / Esc

Printer-friendly Version

Interactive Discussion 


\section{CPD}

10, 1101-1127, 2014

\section{Changing climatic response}

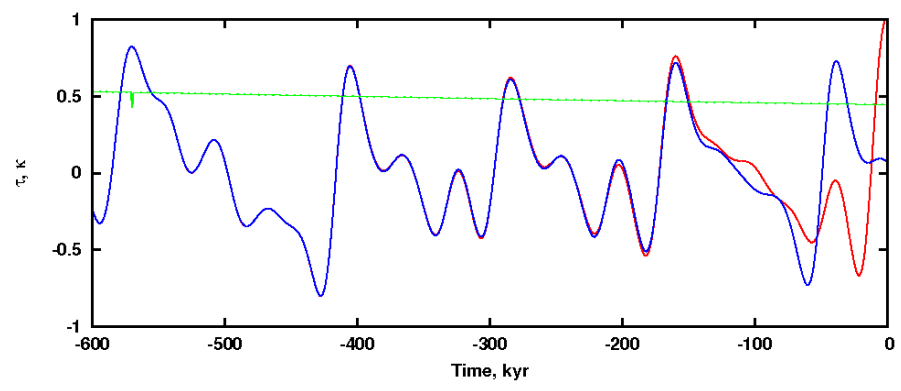

\section{Daruka and}

P. D. Ditlevsen

\section{Title Page}

\section{Abstract}

Introduction

Conclusions

References

Fig. 7. A small perturbation in the climatic damping coefficient (green curve) leads to a remarkable shift of the glacial cycles, constituting a climatic butterfly effect. The blue and red curves correspond to the resulting temperature anomalies without and with the perturbation in the time protocol of the climatic damping coefficient, both driven by real obliquity data (Berger and Loutre, 1991) in the same way as described in the text for Fig. 1. The parameter values are $\lambda=10, \kappa_{0}=1.1, \kappa_{1}=0.3, t_{0}=3.5 \mathrm{Myr}, t_{\mathrm{s}}=2.008 \mathrm{Myr}$, and $x_{0}=-1.2756, \tau_{0}=0$ were used as initial conditions.

Tables

Figures

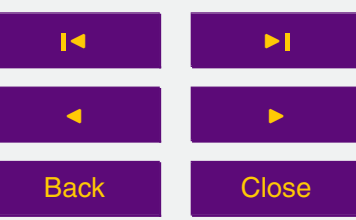

Full Screen / Esc

Printer-friendly Version

Interactive Discussion 


\section{CPD}

10, 1101-1127, 2014
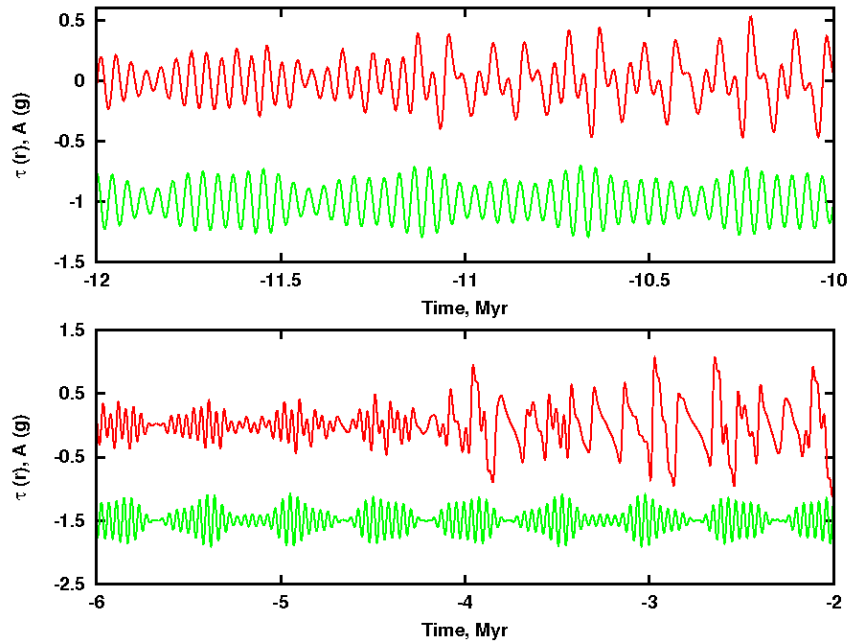

(b)

Title Page

Abstract

Introduction

Conclusions

References

Tables

Figures

Fig. 8. (a) Climatic noise induced MPT. The figure demonstrates the $41 \mathrm{kyr}$ pre-MPT and the $(80+120)$ kyr post-MPT temperature oscillations cycles (red curve). The green curve represents the model external drive Eq. (5), scaled and vertically shifted for better visibility. (b) Obliquity irregularities induced MPT. The figure demonstrates the $41 \mathrm{kyr}$ pre-MPT and the post-MPT "saw-tooth shaped" temperature oscillations (red curve). The green curve represents the model external drive Eq. (5), scaled and vertically shifted for better visibility. The parameter values used for these plots are discussed in the text.

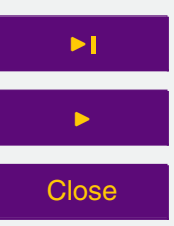

Back

Full Screen / Esc

Printer-friendly Version

Interactive Discussion 